\title{
Three-dimensional simulation of slip-flow and heat transfer in a microchannel using the lattice Boltzmann method
}

\author{
A. C. M. Sousa ${ }^{1,2}$, M. Hadavand ${ }^{1} \&$ A. Nabovati ${ }^{3}$ \\ ${ }^{I}$ Department of Mechanical Engineering, University of New Brunswick, \\ Canada \\ ${ }^{2}$ Department of Mechanical Engineering, University of Aveiro, Portugal \\ ${ }^{3}$ Department of Mechanical \& Industrial Engineering, \\ University of Toronto, Canada
}

\begin{abstract}
In micro- and nano-flows, since the molecular mean free path is comparable to the system's characteristic length, the effect of rarefaction should be considered. In this category of flows, the continuum assumption is no longer valid; therefore, heat transfer, velocity profile and pressure drop markedly and depart from those which are common to macro-flows. Rarefaction may occur in various applications, such as in zero gravity flights and vacuum devices. This phenomenon can occur at the wall surfaces when the Mach number is sufficiently small, which is the case of interest in this study. In these conditions, at the solid-fluid interfaces, the fluid has a slip velocity and a temperature jump is also present. Three-dimensional, thermally developing incompressible laminar flows in a square microchannel with constant temperature walls are studied numerically using the lattice Boltzmann method. The effect of rarefaction on the velocity and temperature distributions and on the Nusselt number is analysed. The LBM predictions are compared with those obtained using the Navier-Stokes equations with the slip condition for the same configuration. The results indicate that an increase of the slip velocity yields an increasing Nusselt number, whereas an increase of the temperature jump has the opposite effect on the Nusselt number values; therefore, the combined result of these two competing effects may yield an increase or decrease on the Nusselt number.
\end{abstract}

Keywords: slip-flow, rarefaction, heat transfer, Knudsen number, lattice Boltzmann method, microchannel. 


\section{Introduction}

In recent years, fluid and heat flow in microchannels has been an emergent and important research area. Its applications are found in a wide range of areas, such as electronic systems, biochemical processes, computer chips, micro-electromechanical systems, optoelectronics, and chemical separations. In micro- and nano-sized channels, one of the major difficulties in the flow prediction is due to the rarefaction effects that occur when the channel dimensions are comparable to the mean free path of the fluid molecules [1]. The continuum assumption is no longer valid and the gas exhibits non-continuum effects, such as velocity slip and temperature jump, at the channel walls.

The Knudsen number, $K n$, which is defined as the ratio of the lattice molecular mean free path, $\lambda$, to the characteristic length of the system [2], indicates the deviation of the fluids from their continuum behaviour. As $K n$ increases, the effect of rarefaction becomes dominant, which influences the shear stress, velocity profile, pressure drop, and heat flux. For small Knudsen numbers $\left(K n \leq 10^{-3}\right)$, the flow can be assumed to be continuous with no-slip boundary condition on the solid walls. For large values of $K n(K n \geq 10)$, free molecular flow is assumed and the occurrence of intermolecular collisions is negligible compared to that of the collisions between the gas molecules and the walls. For intermediate values of $K n(0.1<K n<10)$, a transition flow regime takes place, and the continuum assumption required to use the Navier-Stokes equations is no longer valid. However, the intermolecular collisions are not yet negligible and should be taken into account in the flow field. In the range $0.001<K n<0.1$, slip flow is the representative flow regime. The Navier-Stokes equations combined with the energy equation are usually employed for flows in this regime, but not for values of $K n$ larger than 0.1. Several numerical and experimental studies on this topic have been conducted using the Navier-Stokes equations [1, 3-6].

For microflow simulations, molecular dynamics (MD) [7], and the direct simulation Monte Carlo method (DSMC) [8] are popular because the approach is valid for the full range of flow regimes (from continuum to free molecular). However, DSMC simulations are computationally intensive and provide large microscopic databases, which, in general, are excessive for most of the practical applications.

The lattice Boltzmann method (LBM) is a relatively new discrete particlebased mesoscopic approach for Computational Fluid Dynamics, which has the potential to improve the above-mentioned situation because it is efficient, and its solutions are comparable to those of the Navier-Stokes solvers, primarily because it can recover the Navier-Stokes equations. The fundamental idea behind LBM is to construct the simplified kinetic models that capture the essential physics of microscopic behaviour so that the macroscopic flow description, built upon the quantities at microscopic level, obey the desired continuum equations of fluid dynamics. Thus, LBM is based on the particle dynamics governed by a simplified model of the Boltzmann equation; the simplification is usually introduced into the nonlinear collision integral. This method is suitable for solving the low- speed rarefied gas flows in the whole flow regime. 
Nie et al. [9] simulated a 2D-microchannel flow with the bounce-back boundary treatment. Lim et al. [10] simulated two-dimensional microchannel flow and obtained good results by employing the specular bounce-back rule and the extrapolation scheme for slip velocity. Succi [11] showed that a slip velocity on the wall can be captured by using a combination of the bounce-back and specular-reflection conditions. Jeong et al. [12] studied the compressibility and rarefaction of a pressure-driven gas in three- dimensional (3D) microchannels by using LBM; their results are in good agreement with some 2D limiting cases. Chew et al. [13] presented a three-dimensional lattice Boltzmann BGK (Bhatnagar-Gross-Krook) model for simulating micro flow with heat transfer. The 3D model they used was an extension of the two-dimensional model which was based on the classic kinetic theory and the thermal lattice Boltzmann method (TLBM). They applied the Taylor series expansion and least square-based LBM (TLLBM) to simulate microflow and heat transfer in rectangular microchannels.

In this study, the aim is to show the ability of the three dimensional lattice Boltzmann method with the single relaxation BGK approximation to simulate microflow with heat transfer. The effect of rarefaction and Peclet number $(\mathrm{Pe})$ on the Nusselt number $(\mathrm{Nu})$ is studied for thermally developing flows in a square microchannel with walls at constant temperature, and the results are compared against the results obtained by using the analytical solution reported by Ebert and Sparrow [14], and the Navier-Stokes equations [1].

\section{Mathematical model}

The thermal lattice Boltzmann model utilizes two distribution functions, $f$ and $g$, for the flow field and the temperature field, respectively. The $f$ and $g$ functions have directional components on each lattice site; each direction-wise component represents the probability of finding fluid or thermal particles on that lattice site, at a specific time, and in the assigned direction. The two distribution functions obey their respective lattice Boltzmann transport equations with the single relaxation BGK approximation [16]. For the flow field and the temperature field, those are given as follows:

$$
\begin{aligned}
& f_{i}\left(\vec{x}+c_{i} \delta t, t+\delta t\right)-f_{i}(\vec{x}, t)=-\frac{\delta t}{\tau_{v}}\left[f_{i}(\vec{x}, t)-f_{i}^{e q}(\vec{x}, t)\right] \\
& g_{i}\left(\vec{x}+c_{i} \delta t, t+\delta t\right)-g_{i}(\vec{x}, t)=-\frac{\delta t}{\tau_{c}}\left[g_{i}(\vec{x}, t)-g_{i}^{e q}(\vec{x}, t)\right]
\end{aligned}
$$

where $\delta x$ and $\delta t$ are the lattice grid spacing and the time step, respectively; $c_{i}$ is the particle discrete velocity and $c_{s}$ is the speed of sound which is related to streaming speed by $c_{s}=c / \sqrt{3} . \tau_{v}$ and $\tau_{c}$ are the momentum and internal energy relaxation times, respectively.

The present simulations are based on the D3Q19 model. For this model, the discrete velocity set is given as: 
78 Advanced Computational Methods and Experiments in Heat Transfer XI

$$
c_{i}= \begin{cases}0 & i=0 \\ ( \pm 1,0,0),(0, \pm 1,0),(0,0, \pm 1) & i=1-6 \\ ( \pm 1, \pm 1,0),( \pm 1,0, \pm 1),(0, \pm 1, \pm 1) & i=7-18\end{cases}
$$

$f_{i}$ and $g_{i}$ are the density distribution function and internal energy density distribution function, respectively; $f_{i}^{e q}$ and $g_{i}^{e q}$ are their corresponding equilibrium distribution functions, which are formulated as follow:

$$
\begin{gathered}
f_{i}^{e q}=\rho \omega_{i}\left[1+\frac{1}{c_{s}^{2}} c_{i} \cdot u+\frac{1}{2 c_{s}^{4}}\left(c_{i} \cdot u\right)^{2}-\frac{1}{2 c_{s}^{2}} u \cdot u\right] \\
g_{i}^{e q}=\omega_{i} T\left[1+\frac{1}{c_{s}^{2}} c_{i} \cdot u+\frac{1}{2 c_{s}^{4}}\left(c_{i} \cdot u\right)^{2}-\frac{1}{2 c_{s}^{2}} u \cdot u\right]
\end{gathered}
$$

where $\omega_{i}$ is the weighing factor, which is defined for the three-dimensional lattice with 19 velocities as:

$$
\omega_{i}= \begin{cases}1 / 3 & i=0 \\ 1 / 18 & i=1-6 \\ 1 / 36 & i=7-18\end{cases}
$$

The macroscopic velocity $u$, density $\rho$, pressure $P$, and temperature, $T$ are calculated as follows:

$$
\rho \mathbf{u}_{\mathbf{i}}=\sum_{i} c_{i} f_{i}, \quad \rho=\sum_{i} f_{i}, \quad P=\rho c_{s}^{2}, \quad T=\sum_{i} g_{i}
$$

In order to simulate the microflow using the lattice Boltzmann method, correlating the parameter $\tau_{v}$ with $K n$ is important. Lim et al. [10], assumed the gas molecules travel the distance of the lattice mean free path $l$ with the lattice speed $c=\delta x / \delta t$. With this assumption, the $K n$ can be defined as:

$$
K n=\frac{l}{H}=\frac{\delta x \tau_{v}}{H}
$$

where $H$ is the channel height in the present study. Arkilic et al. [17] calculated the slip velocity boundary condition by assuming the slip velocity is proportional to the shear rate at the wall. The slip velocity condition and the Maxwell firstorder temperature jump boundary condition are given as follows: 


$$
\begin{gathered}
\left.U\right|_{\text {wall }}=\frac{\left(2-\sigma_{v}\right)}{\sigma_{v}} K n \frac{\partial U}{\partial n} \\
T-\left.T\right|_{\text {wall }}=\frac{\left(2-\sigma_{T}\right)}{\sigma_{T}}\left(\frac{2 \gamma}{\gamma+1}\right) \frac{K n}{\operatorname{Pr}} \frac{\partial T}{\partial n}
\end{gathered}
$$

where $U, T$, and $n$ are non-dimensional velocity, non-dimensional temperature, and the wall normal coordinate; $\gamma$ is the specific heat ratio and $\operatorname{Pr}$ is the Prandtl number; $\sigma_{v}$ and $\sigma_{T}$ are the tangential momentum and energy accommodation coefficients, which depend on the working fluid properties, the surface material and its finishing. These two coefficients are usually set equal to 1.0, which means that at the wall boundary only fully diffuse reflection does occur. The temperature jump boundary condition can be written in the following form:

$$
T-\left.T\right|_{\text {wall }}=\frac{K n}{\beta} \frac{\partial T}{\partial n}
$$

where the parameter $\beta$ is a function of the Prandtl number and the specific heat ratio, namely: $\beta=\left(\frac{\gamma+1}{2 \gamma}\right) \operatorname{Pr}$.

The Prandtl number is calculated based on the following relation:

$$
\operatorname{Pr}=\frac{\tau_{v}-0.5}{\tau_{c}-0.5}
$$

\section{Results and discussion}

The three dimensional thermal lattice Boltzmann model is employed to predict the flow field and heat transfer in a microchannel with a square cross-section. The fully developed flow along the channel length with average velocity $u_{\text {ave }} i s$ considered for this study. The non-dimensional wall temperature and inlet temperature are set to one and zero, respectively. The schematic of the square microchannel along with the coordinate system considered in this study is shown in Figure 1.

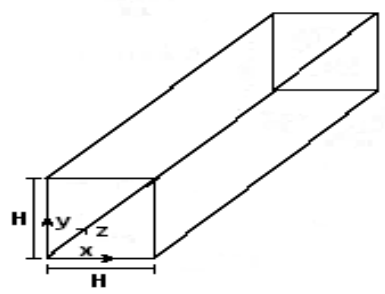

Figure 1: Schematic diagram of the square microchannel. 




Figure 2: Comparison of the predicted fully developed velocity profile along the channel central plane $(x / H=0.5)$ with the analytical results of Ebert and Sparrow [14].

To examine the independence of the numerical solution from the domain size, several domain sizes were investigated, and the domain size of $50 \times 50 \times 800$ lattice units was found to be of adequate resolution. The outlet boundary is placed far enough, so that the results are not affected by the outlet boundary condition.

The fully developed velocity profiles along the channel central plane $(x / H=0.5$ or $y / H=0.5)$ for different Knudsen numbers are reported in Figure 2. The slip velocity increases with increasing Knudsen number. The present results, when compared with the analytical results of Ebert and Sparrow [14], indicate very good agreement, as shown in Figure 2.

The effect of the $K n$ and $\beta$ on the temperature profile is shown in Figure 3 for thermally developing flow at $P e=1$, which $P e$ is the Peclet number, $P e=R e P r$. For no-slip-flow condition, $K n=0$, there is no temperature jump at the wall-fluid interface. As slip increases, the effect of $K n$ and $\beta$ on the temperature profile becomes apparent. For low $\beta$ values, the temperature profile deviates noticeably from the $K n=0$ case due to large temperature jump at the walls. For large $\beta$, the temperature jump at the wall decreases and the temperature profile follows closely to no-slip flow. $Z^{*}$ is the reciprocal Graetz number, which is given by $z /(H \operatorname{Pr} R e)$. 

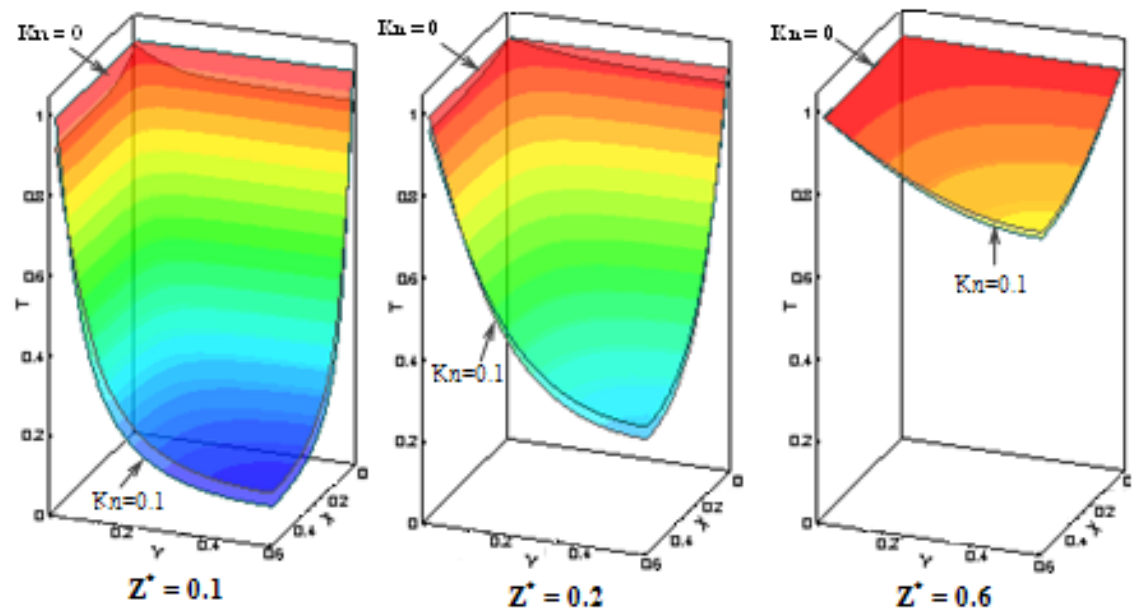

a) $\beta=10$
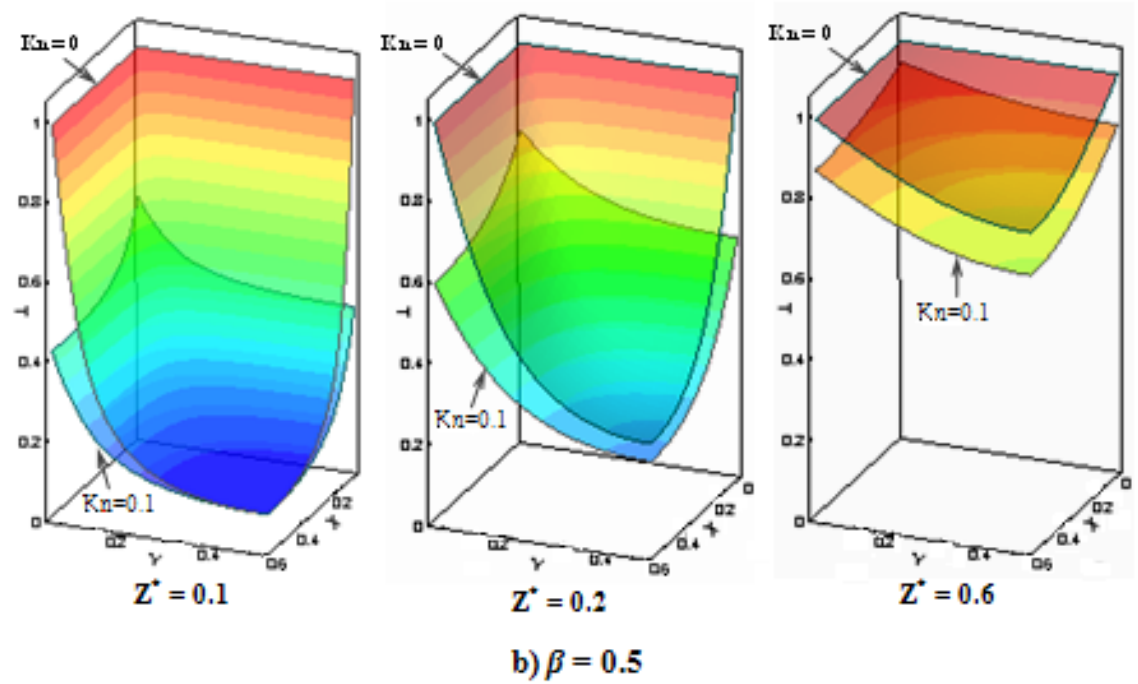

Figure 3: Effect of rarefaction on the thermally developing temperature profile at different cross-sections along the length of a square microchannel for $P e=1$ and $K n=0$ and 0.1 at (a) $\beta=10$ and (b) $\beta$ $=0.5$.

The effect of the rarefaction on the thermally developing temperature profile at different central planes along the length of the square microchannel for $P e=1$ is shown in the Figure 4. The slip temperature decreases with increasing Knudsen number. The average fluid temperature at every cross-section along the channel decreases with increasing Knudsen number which causes a higher thermal entrance length. 
82 Advanced Computational Methods and Experiments in Heat Transfer XI

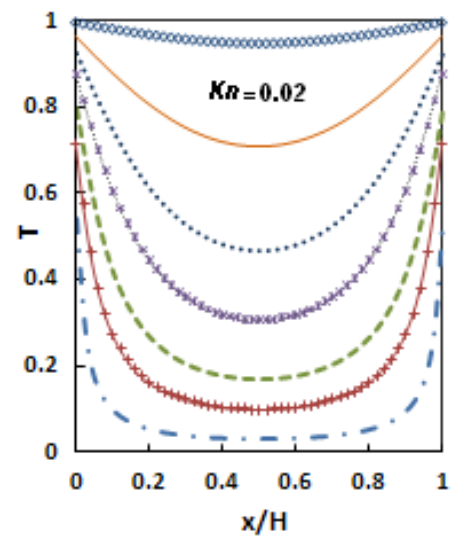

a



b

Figure 4: Fluid temperature profile at the central plane $y / H=0.5$ along the length of the square microchannel for $P e=1$ and $\beta=0$ at (a) $K n=0.02$ and (b) $K n=0.1$.

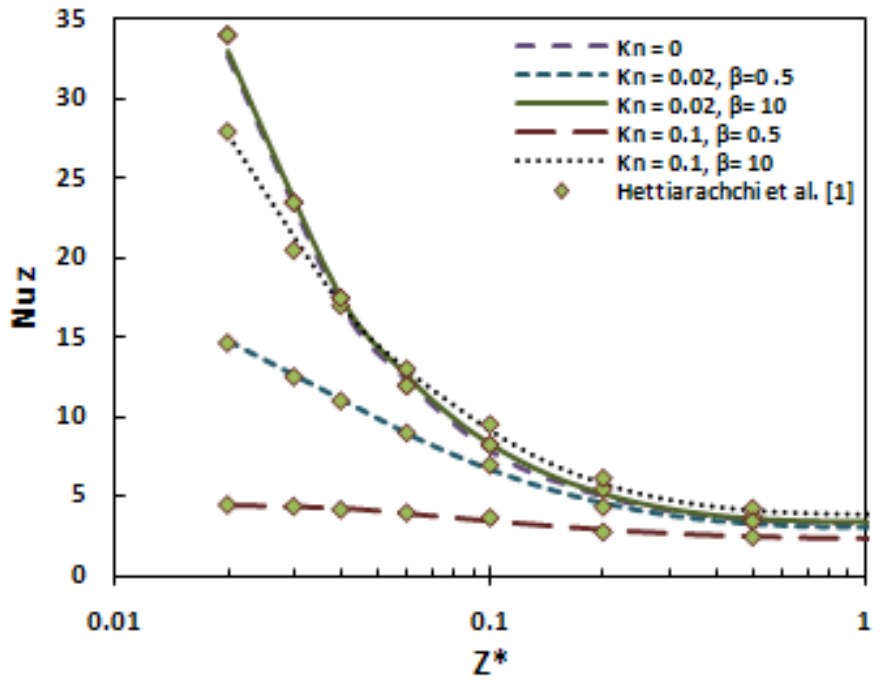

Figure 5: Comparison of the effect of $K n$ and $\beta$ on the developing local Nusselt number along the axis of a square microchannel with the results using the Navier-Stokes equations [1] for $P e=1$. 


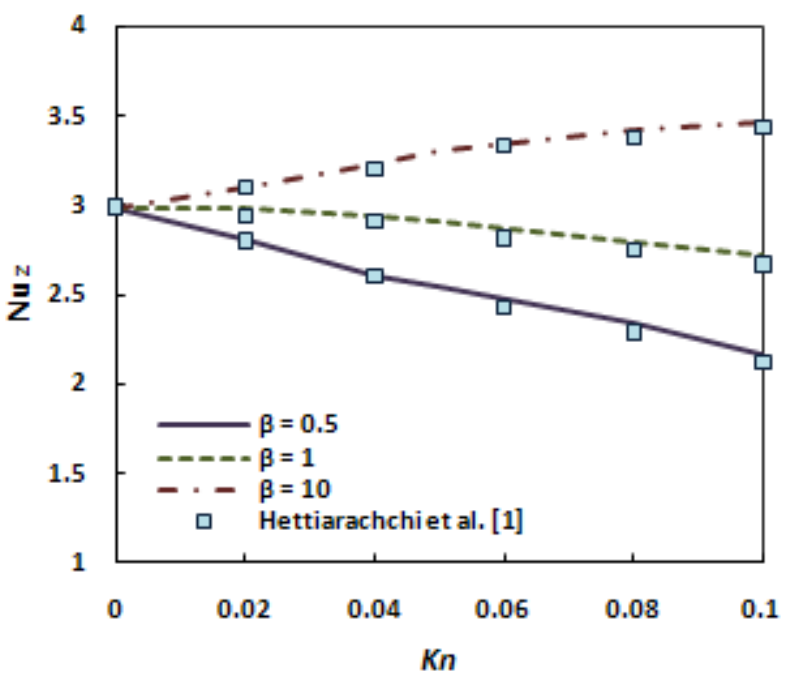

Figure 6: Comparison of the results of fully developed Nusselt number with the results using the Navier-Stokes equations [1] for different values of $\beta$.

Figure 5 shows the effect of the $K n$ and $\beta$ on the local Nusselt number in the entrance region for $P e=1$. The drop in Nusselt number early in the entrance region for all cases is in agreement with the findings of Navier-Stokes based model reported by Hettiarachchi et al. [1]. The effect of $\beta$ on $N u$ is dominant right at the entrance. For lower values of $\beta$, a large temperature jump occurs (Figure 3) at the beginning of the entrance region which results in a drop in the Nusselt number. In the case of slip-flow, the presence of the velocity slip and temperature jump can significantly affect the local Nusselt number. The velocity slip increases the advection near the walls causing an increase in heat transfer rate; however, the temperature-jump increases the thermal resistance at the wallfluid interface, which yields a decrease in the heat transfer rate.

The variation of fully developed Nusselt number with $K n$ and $\beta$ is shown in Figure 6 for lower values of $\beta$; the deviation of the average Nusselt number for higher $K n$ numbers from the no-slip boundary condition case is evident in Figure 6. As mentioned above, this happens mostly due to the dominant effect of the temperature jump on the Nusselt number.

\section{Conclusion}

In this work, rarefaction effects on the fluid flow and heat transfer in microchannels of square cross section with constant wall temperature were investigated using the BGK lattice Boltzmann method. The slip and jump boundary conditions at the wall were obtained analytically with a first order in $K n$ derivation based on the kinetic theory. The numerical predictions using this 
method compare well with those obtained using the Navier-Stokes equations by Hettiarachchi et al. [1]. The rarefaction effect reduces the maximum velocity in the channel. The wall temperature jump and slip velocity of fluid on the microchannel wall increases as the $K n$ increases, and the entrance length increases with the increase of $K n$.

The Nusselt number increases with increasing slip velocity but an increase of the temperature jump has the opposite effect on the Nusselt number, so the combined result of these two competing effects may yield an increase or decrease of the Nusselt number. For the range $0.02<K n<0.1$, the variation of the fully developed Nusselt number goes from a 15\% increase to around a $30 \%$ decrease, when compared to the case with no slip boundary condition.

These results clearly indicate the lattice Boltzmann method is capable of predicting 3D thermal problems with slip boundary conditions.

\section{Acknowledgement}

The authors acknowledge the support received from NSERC (Natural Sciences and Engineering Research Council of Canada) through the Discovery Grant 12875 (ACMS).

\section{References}

[1] Hettiarachchi, H.D.M., Golubovic, M., Worek, W.M., \& Minkowycz, W.J., Three-dimensional laminar slip-flow and heat transfer in a rectangular microchannel with constant wall temperature. International Journal of Heat and Mass Transfer, 51, pp. 5088-5096, 2008.

[2] Schaaf, S.A. \& Chambre, P.L., Flow of Rarefied Gases, Princeton University Press, Princeton, NJ, 1961.

[3] Wu, P.Y., \& Little, W.A., Measurement of the heat transfer characteristics of gas flow in one channel heat exchangers for micro miniature refrigerators. Cryogenics, 24, pp. 415-420, 1984.

[4] Cercignani, C. \& Daneri A., Flow of a rarefied gas between two parallel plates. Journal of Applied Physics, 34(12), pp. 3509-, 1963.

[5] Yu, S., \& Ameel, T. A., Slip flow heat transfer in rectangular microchannels. International Journal of Heat and Mass Transfer, 44, pp. 4225-4234, 2001.

[6] Kuddusi L., Prediction of temperature distribution and Nusselt number in rectangular microchannels at wall slip condition for all versions of constant wall temperature. International Journal of Thermal Science, 10 (46), pp. 998-1010, 2007.

[7] Koplik, J., \& Banava, J.R., Continuum deductions from molecular hydrodynamics. Annual Review of Fluid Mechanics, 27, pp. 257-292, 1997.

[8] Bird, G.A., Molecular Gas Dynamics and the Direct Simulation of Gas Flows, Oxford: Clarendon, 1994. 
[9] Nie, X., Doolen, G.D., \& Chen, S.Y., Lattice Boltzmann simulation of fluid flows in MEMS. Journal of Statistical Physics, 107, pp. 279-89, 2002.

[10] Lim, C.Y., Shu, C., Niu, X.D., \& Chew, Y.T., Application of lattice Boltzmann method to simulate microchannel flows. Physics of Fluids, 14, pp. 2299-2308, 2002.

[11] Succi, S., Mesoscopic modeling of slip motion at fluid-solid interfaces with heterogeneous catalysis. Physical Review Letter, 89, 064502, 2002.

[12] Jeong, N., Lin, C.L., \& Choi, D.H., Lattice Boltzmann study of threedimensional gas microchannel flows. Journal of Micromechanics and Microengineering, 16, pp. 1749-1759, 2006.

[13] Chew, Y.T., Niu, X.D., \& Shu, C., Three-dimensional lattice Boltzmann BGK model and its application to flows with heat transfer in a rectangular microchannel. International Journal for Numerical Methods in Fluids, 50, pp. 1321-1334, 2006.

[14] Ebert W.A., \& Sparrow E.M., Slip flow in rectangular and annular ducts. Journal of Basic Engineering, 87(4), pp. 1018-1024, 1965.

[15] He, X.Y., Chen, S.Y., \& Doolen, G.D., A novel thermal model for the lattice Boltzmann method in incompressible limit. Journal of Computational Physics, 146, pp. 282-300, 1998.

[16] Bhatnagar, P.L., Gross, E.P., \& Krook, M., A model for collision process in gases. I. Small amplitude processes in charged and neutral one-component system. Physical Review, 94, pp. 511-525, 1954.

[17] Arkilic, E.B., Schmidt, M.A., \& Breuer, K.S., Gaseous slip flow in long microchannels. Journal of Microelectromechanical Systems, 6, pp. 167178, 1997. 